\title{
La biblioteca como gestora y creadora de productos para el control y acceso a la información del Instituto de Ciencias Nucleares, UNAM
}

The library as manager and creator of products for controlling and accessing the information of the Institute of Nuclear Sciences, UNAM

\author{
María Magdalena SIERRA FLORES \\ Unidad de Información y Biblioteca, Instituto de Ciencias Nucleares, UNAM, Mexico, sierra@nucleares.unam.mx
}

\begin{abstract}
Resumen
Se presenta el proyecto realizado por la Unidad de Información y Biblioteca (UIB) del Instituto de Ciencias Nucleares (ICN) de la UNAM sobre el desarrollo de un Sistema Integral de Gestión de Información (SIGI). Como base del proyecto de automatización se tomó el informe anual de actividades, elaborado por los académicos de la institución. Se describe el diseño, las actividades y productos de información, particularmente la generación de indicadores bibliométricos institucionales, el directorio académico, y el repositorio institucional, así como productos que describen el quehacer cotidiano de la comunidad. La biblioteca ha pasado de un rol tradicional a uno nuevo en el que resulta clave como gestor de la información.
\end{abstract}

Palabras clave: Gestión de la información. Gestión de documentos. Estudios de caso. Sistema y productos. Instituto de Ciencias Nucleares (UNAM, México).

\section{Introducción}

Este artículo muestra cómo los bibliotecarios pueden innovar y crear herramientas y productos que apoyen la toma de decisiones. Si los bibliotecarios son capaces de brindar servicios especializados y elaborar bibliografías, perfiles de interés para ofrecer el servicio de diseminación selectiva de la información, desarrollar colecciones y organizarlas; entonces pueden aventurarse en los productos de información generados en sus instituciones, analizar sus contenidos y crear productos útiles para apoyarla y apoyarse en la labor de cada día.

El fin del artículo es mostrar de manera general los procesos realizados para la transformación de una biblioteca tradicional en otra gestora de la información. Se describen en forma breve y general los pasos realizados para llegar a obtener los cambios y productos planeados. Quienes se aventuren a convertirse en los gestores de la información, podrán tener en el presente trabajo un ejemplo o caso particular.

\begin{abstract}
The project of the Library and Information Unit (UIB) of the Institute of Nuclear Sciences (ICN) of the UNAM to develop an integrated information management system, called SIGI, for auditing its academic resources and production is presented. As the basis of the automation project, the library took the annual activity report drawn up by the academics of the institution. The processes of change and design, and the resulting activities and information products are described, in particular, the generation of institutional bibliometric indicators, the academic directory and the institutional repository. The library has evolved from a traditional role to a new one in which it has become a scientific information management unit.
\end{abstract}

Keywords: Information management. Document management. Case studies. System and products. Institute of Nuclear Science, UNAM.

Hace una década el Instituto de Ciencias Nucleares de la UNAM (ICN, UNAM) carecía de una herramienta capaz de albergar la información producida por su comunidad académica,tanto para su organización, control y gestión, como para promoverla y divulgarla entre las comunidades afines y de interés.

Por otro lado, las constantes demandas de información derivadas de la actividad académica por parte de instancias tanto internas como externas a la UNAM, se convertían en actividades tediosas que consumían mucho tiempo.

Conscientes de la problemática y con el objetivo de brindar una solución y apoyar en el control y manejo de la información, la Unidad de Información y Biblioteca (UIB) del ICN presentó un proyecto que planteaba el desarrollo de un sistema de información del que derivarán diversos productos que permitieran dar respuesta a las constantes demandas realizadas a la institución así como a la propia UIB.

Actualmente y después de una lucha constante por crear la herramienta idónea, se cuenta con un sistema de información denominado Sistema 
Integral de Gestión de Información (SIGI). Esta herramienta genera distintos productos diseñados a la medida de cada demanda, entre ellos el informe anual de actividad a nivel departamental e institucional, estadísticas para apoyar la planeación y un directorio académico. Es importante señalar que la información base del SIGI la constituye el informe de actividades que la comunidad académica reporta anualmente.

Una vez atendidas las demandas de información a nivel institucional y conociendo la riqueza informativa del SIGI, se comezó el desarrollo de productos con valor agregado derivados de los primeros, entre éstos destacan los indicadores bibliométricos, un repositorio institucional y los contenidos que describen la actividad académica de la institución incluida en su página web. Esta labor ubica a la UIB como la gestora de la información en el ICN. Además de organizar los informes anuales de actividad, se crearon diversos productos que le han permitido ofrecer nuevos servicios.

El presente artículo muestra la inserción y participación de la bilioteca en la gestión de la información, así como, los procesos y productos derivados del sistema de información diseñado, el cual involucra la transformación de los procedimientos, recursos, comportamiento y actitud de la comunidad (académicos y bibliotecarios profesionales) a partir de la elaboración del informe anual de actividad.

Para ubicar a la UIB en su contexto institucional así como en la comunidad a la que ofrece sus servicios se describe a continuación el ICN de manera somera.

\section{El Instituto de Ciencias Nucleares}

El Instituto de Ciencias Nucleares (ICN) de la UNAM tiene su origen en 1967. Creado como Laboratorio Nuclear, cinco años después (1972) fue constituido como Centro de Estudios Nucleares (CEN). Gracias a la creciente productividad de la planta académica, en 1988 el CEN fue promovido a Instituto de Ciencias Nucleares (ICN). Sus áreas de especialidad se enfocan a la investigación teórica, experimental y aplicada en diversas áreas de la física y la química sobre la materia y sus interacciones (Alcubierre, 2013). Su misión es:

1. Realizar investigación teórica y experimental, básica y aplicada, en los programas académicos Estructura de la Materia, Física de Altas Energías, Física de Plasmas, Gravitación y Teoría de Campos, Interacción de Radiación con Materia, Química de SItas tempera- turas y Radicales Libres, Química de Plasmas, Química de Radiaciones y Dosimetría.

2. Contribuir a la formación de profesionistas e investigadores, mediante la transmisión de conocimientos a través de cursos y la dirección de trabajos, en los programas de licenciatura y posgrado de la UNAM que son afines a las especialidades del Instituto.

3. Difundir los resultados de las investigaciones que se realizan en el Instituto, mediante la organización, promoción y participación en reuniones de trabajo nacionales e internacionales.

4. Contribuir al desarrollo de las ciencias nucleares, promoviendo los conocimientos generados en las áreas de investigación del instituto (Alcubierre, 2013).

En poco más de cuatro décadas incrementó sus áreas de investigación y modificó su estructura organizacional, pasando de tres a cinco departamentos (los primeros estaban conformados por el de Física y Matemáticas Aplicada, Química y Aplicaciones Nucleares). En 1986 el ICN amplió sus áreas de investigación, por lo que el departamento de Química pasó a conformar al de Química de Radiaciones y Radioquímica y el de Aplicaciones Nucleares pasó al de Gravitación y Teoría de Campos. Finalmente en 1997 se modifica su estructura organizacional conformando cinco departamentos: Estructura de la Materia, Física de Altas Energías, Gravitación y Teoría de Campos, Física de Plasmas y de Interacción de Radiación con Materia y Química de Radiaciones y Radioquímica. Con las modificaciones, su plantilla académica se vio favorecida pasando de los 29 investigadores de 1976 a 61 en 2015.

Los resultados obtenidos en su labor de investigación son publicados en su mayoría en revistas de impacto de sus respectivas áreas, habiendo pasado en los últimos 15 años su promedio de publicación de 2 a 3 artículos por investigador por año. Los resultados también se presentan en reuniones científicas, $u$ otros eventos dirigidos al público en general. Cabe destacar que diversos resultados han sido acreedores a premios y reconocimientos tanto a nivel nacional como internacional.

En cuanto a la formación de recursos humanos, su comunidad académica ha impartido a nivel de licenciatura 614 cursos en el período 20002014. Se participa en tres programas de posgrado de la UNAM - Ciencias Físicas, Ciencias Químicas y Astrofísica-, en los cuales se han ofrecido un total de 700 cursos en el mismo período. Su promedio de alumnos graduados 
por investigador por año pasó de 0,3 a 0,9, es decir, casi un alumno por investigador. Además, recluta a investigadores jóvenes tanto de México como del extranjero para realizar estancias posdoctorales.

En resumen y en palabras de Alcubierre (2013) podemos señalar que el ICN es un activo centro de investigación científica, docencia, difusión, y servicio a la industria, cuyo proceso de maduración a lo largo de cuarenta años le ha permitido tener un impacto significativo en el contexto nacional e internacional.

\subsection{La Unidad de Información y Biblioteca}

La Unidad de Información y Biblioteca (UIB) nace con la creación del entonces Laboratorio Nuclear en la Universidad Nacional Autónoma de México (UNAM). Desde entonces ha ido modificándose y creciendo junto con el Instituto de Ciencias Nucleares. Su objetivo es (Instituto de Ciencias Nucleares, 2009)

Proporcionar y facilitar el acceso a la información, extendiendo el conocimiento en sus diversas líneas de investigación y buscando eliminar las barreras que existen entre el usuario y la información, al facilitar el acceso remoto y simultáneo a libros y documentos, preservar la integridad física de los soportes materiales de la información, además de integrar a la biblioteca a los planes académicos de la Institución, para satisfacer las necesidades de información del personal académico y estudiantes asociados.

En el 2005 se presentó y aprobó ante el Consejo Interno del ICN una propuesta de reestructuración interna en la Unidad de Biblioteca acorde a la misión de la institución y a las actuales necesidades de información de su comunidad académica. Los cambios fueron tanto a nivel organizacional, como tecnológicos, de servicios y culturales. Con el cambio organizacional se logró crear una nueva área (Información) y se constituyó como Unidad de Información y Biblioteca (Figura 1).

Esta estructura organizacional permitió incorporar las actividades relacionadas con el desarrollo de sistemas, la obtención de indicadores bibliométricos y la elaboración de un repositorio institucional. Así mismo, siguió contemplando las actividades propias de la biblioteca, entre éstas el desarrollo de las colecciones a través del proceso de adquisición, las actividades que involucran el análisis bibliográfico y los servicios de información generales y especializados.



Figura 1. Organigrama de la UIB, ICN

En el aspecto tecnológico, se adquirió un servidor, y se redistribuyo el equipo existente (PCs, scanneres e impresoras) y se modificaron congruentemente las instalaciones eléctricas.

En cuanto a los servicios se crearon nuevos y se modificaron otros. El ejemplo más notable es la elaboración del informe anual de actividades, que originalmente se realizaba de forma manual en formato libre; y actualmente se ingresa de manera directa en el sistema (SIGI) en plantillas normalizadas.

Respecto a los cambios culturales que se produjeron en la comunidad académica durante el proceso, destacan los acontecidos en comportamientos y actitudes, con casos a favor y otros en contra del cambio. Con el paso del tiempo y los productos ofrecidos, día a día son más los académicos que participan. Las expectativas, aspiraciones y necesidades van en aumento, lo que obliga a la UIB a mejorar los productos y servicios.

Los cambios realizados en la UIB han tenido una naturaleza sistémica, afectándose entre sí, y produciendo un efecto multiplicador. Se puede decir que se creó un nuevo modelo para la gestión de información.

\subsection{El informe anual de actividad}

\section{Harvey (2006) en distintos estudios}

[... ] confirma la relevancia de los informes escritos como medio para registrar los conocimientos adquiridos en la universidad" e inclusive algunos "permiten aproximarse al nivel de manejo conceptual, textual y lingüístico que se despliega en el ámbito académico, y otros muestran la importancia de los informes como instrumentos para evaluar logros. 
Para el caso del ICN -y seguramente para las instituciones de investigación en general- los contenidos depositados en los informes anuales de actividad incluyen una gran riqueza informativa, que, en su conjunto caracteriza y describe a su comunidad y a la institución (Figura 2). Considerando al informe anual de actividad como uno de los recursos que conduce a la identificación de la actividad cotidiana de la institución, y con la finalidad de concentrarlos para su organización y gestión, se desarrolló el sistema de información con un enfoque integral y relacional, Ilamado Sistema Integral de Gestión de Información (SIGI), con los siguientes objetivos:

- Concentrar la actividad académica del ICN en una fuente, y presentar de manera normalizada el informe anual de actividades para su gestión y difusión.
- Gestionar los datos que proporciona el sistema para optimizar los recursos del instituto.

- Contribuir a la preservación de la producción científica generada en el ICN, a través del diseño de una aplicación informática.

- Facilitar el acceso a la información generada en el ICN a partir de la recopilación, análisis y conversión de los datos que se encuentran dispersos.

- Fomentar la colaboración entre investigadores mediante un sitio interactivo y dinámico capaz de explotar al máximo los recursos que nos brinda la Intranet y sobre todo Internet.

- Dotar de un centro virtual de información de referencia, consultoría y evaluación sobre las áreas de interés del ICN.

\begin{tabular}{|c|c|c|c|c|c|c|}
\hline Datos generales & Datos personales & Formación académica & Datos laborales & & & \\
\hline Líneas de investigación & Líneas & & & & & \\
\hline \multirow{2}{*}{ Producción científica } & Artículos & Artículos en memorias & Edición de memorias & Libros & Capítulos en libros & Patentes \\
\hline & Normas y metodologías & Software desarrollado & Material multimedia & Informes técnicos & Artículos docentes & \\
\hline \multirow[t]{2}{*}{ Docencia y formación de R.H. } & Alumnos de Servicio Social & Tutorías & Dirección de tesis & \begin{tabular}{|l} 
Participación en \\
jurados
\end{tabular} & $\begin{array}{l}\text { Participación en comités } \\
\text { tutorales }\end{array}$ & \\
\hline & Cursos cortos & Cursos formales & Actividad extracurricular & & & \\
\hline Divulgación & Entrevistas en radio y TV & Artículos en periódicos & $\begin{array}{l}\text { Artículos en revistas de } \\
\text { divulgación }\end{array}$ & Folletos & & \\
\hline \multirow{2}{*}{ Actividades Académicas } & Participación en eventos & Organización de eventos & Estancias & Visitas recibidas & $\begin{array}{l}\text { Asociaciones académicas } \\
\text { profesionales }\end{array}$ & \\
\hline & $\begin{array}{l}\text { Participación en comités } \\
\text { editoriales }\end{array}$ & Arbitrajes en revistas & $\begin{array}{l}\text { Participación en órganos } \\
\text { colegiados }\end{array}$ & $\begin{array}{l}\text { Servicios a la } \\
\text { industria }\end{array}$ & & \\
\hline Proyectos & CONACyT & PAPIIT & ICN & Otros & & \\
\hline Logros & Logros destacados & $\begin{array}{l}\text { Reconocimientos, } \\
\text { premios y becas }\end{array}$ & & & & \\
\hline Plan de actividades & Plan 2015 & & & & & \\
\hline Capacitación y actualización & Superación recibida & & & & & \\
\hline Boletines & Calendario & Oficios & & & & \\
\hline
\end{tabular}

Figura 2. Rubros que conforman los contenidos del informe anual de actividad

\section{Metodología}

Las principales acciones realizadas fueron:

1. Crear un equipo de trabajo interdisciplinario: tres bibliotecarios profesionales, dos desarrolladores en sistemas (un bibliotecario y uno de sistemas son externos), académicos (físicos, químicos y astrónomos), además de la participación de los directivos.

2. Identificar y determinar los contenidos de los informes y clasificar por áreas la información reportada (Figura 2).

3. Analizar los requerimientos de información por parte de las distintas instancias, el ICN y por la propia UIB.
4. Elaborar la plantilla que cubra y normalice toda la información.

5. Controlar de forma centralizada la gestión del informe anual de actividades mediante: la programación y desarrollo del Sistema Integral de Gestión de Información (SIGI), la elaboración de procedimientos para la gestión de los informes anuales de actividad, y la capacitación a la comunidad académica en el manejo del SIGI.

6. Diseñar la interface para acceder al SIGI.

7. Implementar el sistema.

8. Analizar y gestionar la información concentrada de los informes individuales. 
9. Determinar los datos para elaborar distintos productos (Cuadro I).

10.Elaborar los productos de información: anexos para el informe institucional (contenidos que describen la actividad académica de la institución), reportes por departamento, estadísticas de planeación y directorio científico.

11. Analizar y gestionar la información reportada en los productos.

12. Elaborar nuevos productos con valor añadido: indicadores bibliométricos, repositorio institucional, selección de productos para su difusión, y diseño de una interface para acceder desde la página web de la biblioteca a los productos desarrollados.

\section{Resultados}

Actualmente el ICN cuenta con un sistema integral y relacional llamado Sistema Integral de Gestión de Información (SIGI), desarrollado en la UIB. Con la intención de distribuir cargas de trabajo, así como responsabilidades, se capacitó a la comunidad académica del ICN en el uso del sistema (SIGI). A la fecha, esta comunidad es responsable de ingresar anualmente su informe de actividades, labor que viene realizando en los últimos 10 años (2005 a 2014).

En la primera etapa de la gestión de los informes anuales, se obtuvieron cuatro productos (Figura 3). También se diseñaron nuevos productos con valor añadido derivados de los primeros (Figura 4).

1. Actividades académicas: describen la labor diaria de la comunidad. El sistema muestra los cursos que imparte cada académico por nivel y área, sus líneas actuales de investigación, publicaciones recientes, eventos organizados, revistas donde participan como árbitros, así como los premios que han recibido (Figura 5).

2. ICN en cifras: con base en los datos estadísticos obtenidos en los informes, se generaron los indicadores bibliométricos, mismos que describen con mayor precisión la actividad académica del ICN (Figura 6).

3. Académicos del ICN: muestra la página personal por académico organizada en diez grandes rubros (descritos de manera general en la Tabla I y Figura 7).
4. Repositorio institucional: contiene la producción científica (detallada en la Tabla I) reportada en los informes.

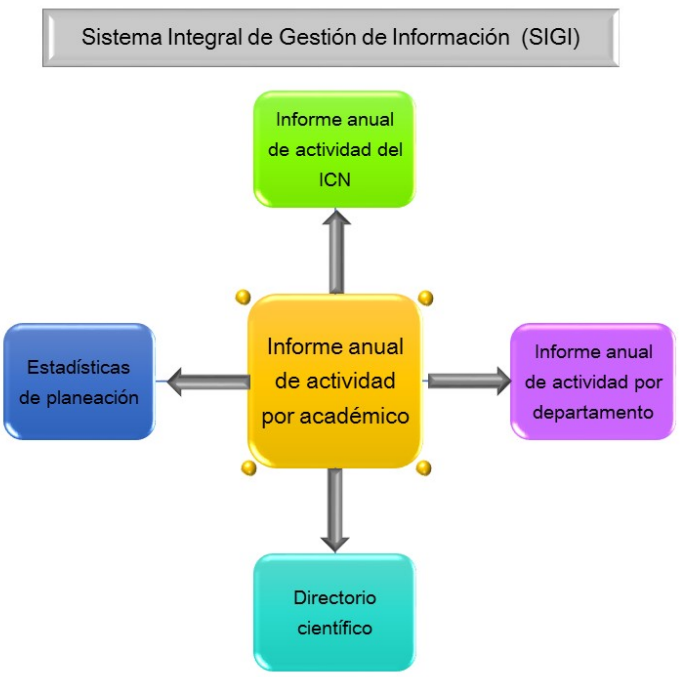

Figura 3. Productos derivados del informe gestionados con SIGI

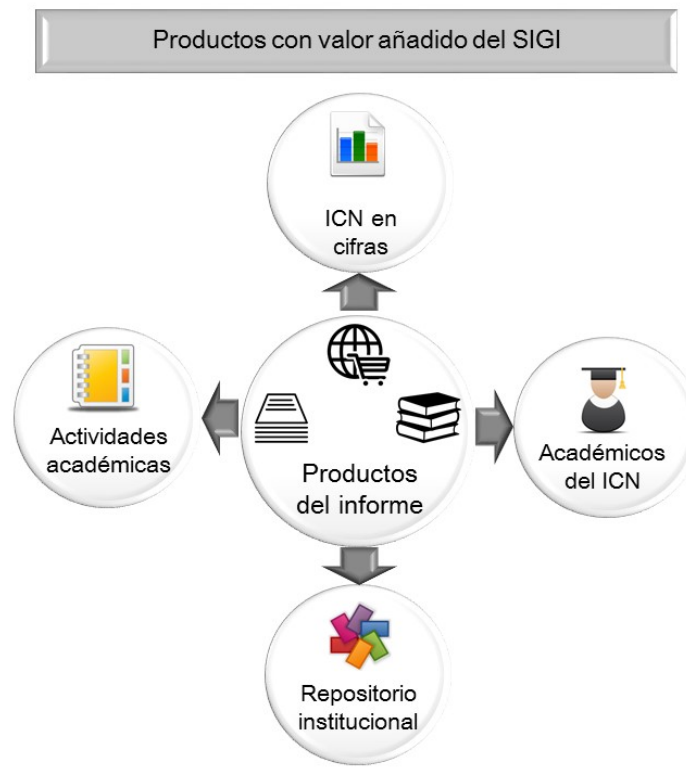

Figura 4. Productos con valor añadido

Finalmente, para promover y divulgar los productos obtenidos se han utilizado las paginas web institucional y de la propia UIB (Figura 8). 


\begin{tabular}{|c|c|c|c|}
\hline Rubros considerados para la obtención de productos & Directorio Indicadores & Actividades & Repositorio \\
\hline \multicolumn{4}{|l|}{ Datos generales } \\
\hline \multicolumn{4}{|l|}{ Líneas de investigación } \\
\hline \multicolumn{4}{|l|}{ Producción científica } \\
\hline \multicolumn{4}{|l|}{ Docencia y formación de recursos humanos } \\
\hline \multicolumn{4}{|l|}{ Actividades académicas } \\
\hline \multicolumn{4}{|l|}{ Proyectos } \\
\hline Premios, distinciones y Reconocimientos & & & \\
\hline
\end{tabular}

Tabla I. Análisis y gestión de la información obtenida de los informes e información utilizada en cada producto


\section{Cursos de Física a nivel licenciatura}


Figura 5. Actividades académicas

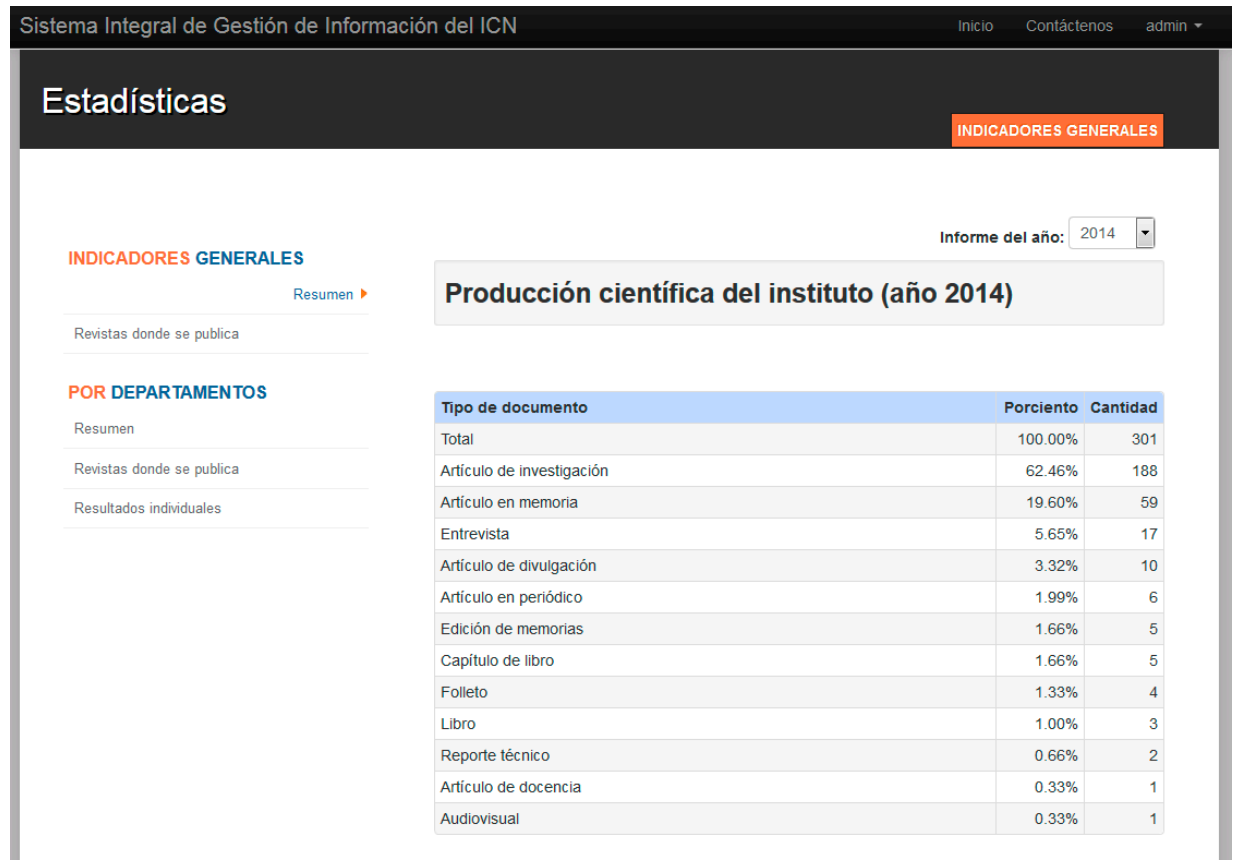

Figura 6. Otros indicadores obtenidos de los datos estadisticos generales 


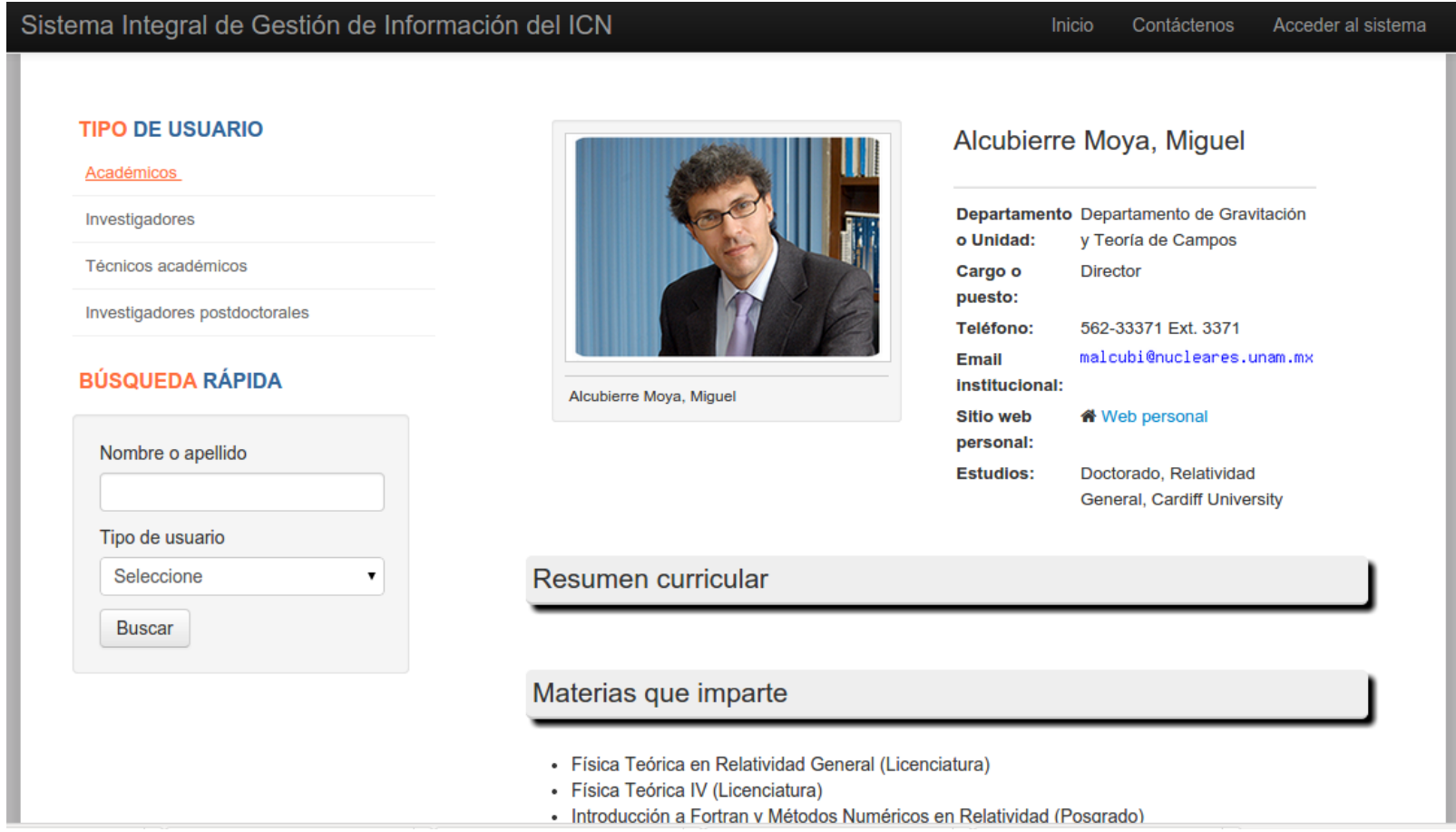

Figura 7. Página personal por académico

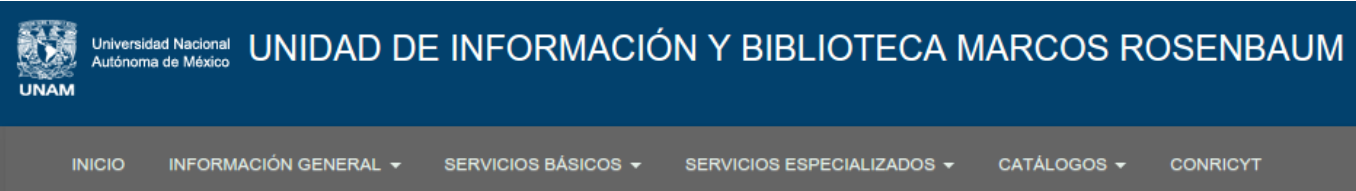

\begin{tabular}{|l|}
\hline e-recursos \\
\hline Revistas \\
\hline Libros \\
\hline Nuevas adquisiciones \\
\hline Material impreso \\
\hline Material electrónico \\
\hline Biblioteca en cifras \\
\hline Los 100 libros mas prestados \\
\hline Áreas del acervo \\
\hline Libros adquiridos por \\
departamento en 2014
\end{tabular}
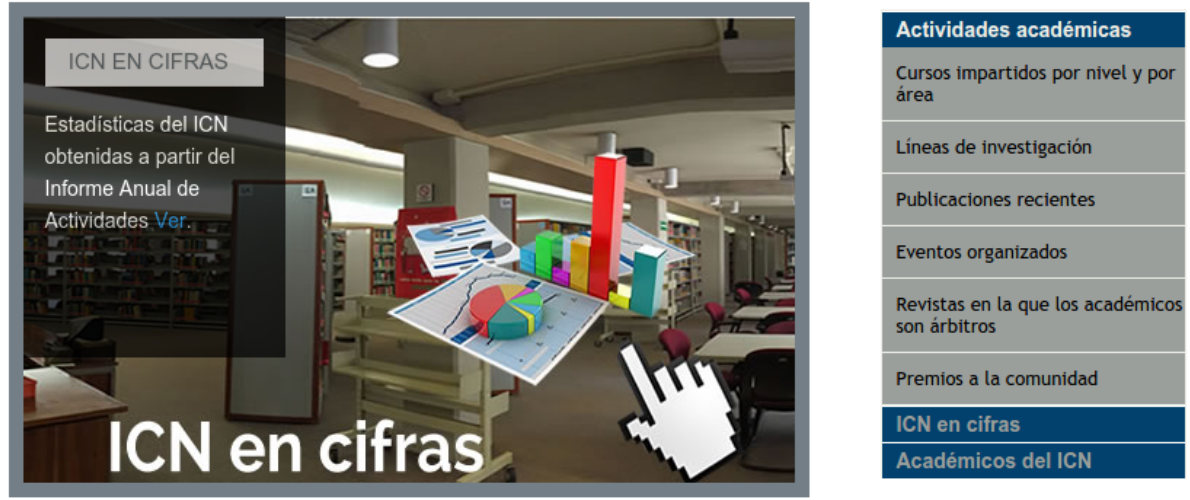

Figura 8. Página Web de la Unidad de Información y Biblioteca

\section{Conclusiones}

Este artículo ha mostrado cómo la biblioteca puede y debe ofrecer productos y servicios de acuerdo a la demanda de su comunidad. En este caso se atendieron servicios específicos para la comunidad y directivos o cuerpo técnico y político de una organización dedicada a la investigación y a la educación superior.

Considerando las recomendaciones de Quijano (2010), en la UIB nos apropiamos de la tecnología de la información (hardware y software), desarrollamos nuevas competencias tecnológicas de información y comunicación, y transferimos los resultados a nuestra comunidad aca- 
démica. Así mismo, percibimos y observamos con mayor profundidad la información reportada en el informe anual de actividad, concebimos las ideas, las cifras e imágenes en el interior y las relacionamos con otros productos, es decir, miramos a lo esencial: los contenidos (Cubillo, 1999).

Gracias a esta acción se logró unificar el informe anual de actividades, así como generar productos con valor agregado que apoyan a la gestión de la información del instituto y de la propia UIB; facilitamos el acceso a la información actualizada en los temas de interés del ICN, y desarrollamos productos que apoyan a la difusión y divulgación de su labor de manera individual e institucional.

También dotamos al Instituto de una herramienta para la gestión de la información. Creamos un espacio virtual dirigido tanto a los académicos como al personal vinculado para disponer de la información, contactos y recursos electrónicos dispersos en la Web. Abrimos una ventana para fomentar la colaboración entre investigadores y acercar la información a la comunidad interesada. En definitiva, en la UIB dimos un salto cualitativo y nos convertimos en un actor central, ahora potenciado, en la gestión de los contenidos.

Finalmente consideramos que los proyectos de este tipo podrían contribuir a la solución de las problemáticas relacionadas con la divulgación de los trabajos científicos, fomento de la investigación, gestión y control de la información generada en las instituciones académicas de investigación.

\section{Referencias}

Aja Quiroga, Lourdes. Gestión de información, gestión del conocimiento y gestión de la calidad en las organizaciones. // ACIMED, Ciudad de La Habana. 10:5 (oct. 2002). http://scielo.sld.cu/scielo.php?script=sci_arttext\&pid=S10 24- 94352002000500004\&lng=es\&nrm=iso (2015-03-18)

Alcubierre Moya, Miguel (2013). Informe de actividades del Instituto de Ciencias Nucleares, UNAM: 2012-2013. México: Universidad Nacional Autónoma de México, Instituto de Ciencias Nucleares, 2013.

Arquero Avilés, Rosario; Cuenca, Gonzalo Marco; Cobo Serrano, Silvia; Ramos Simón, L. Fernando (2014). Comunidades de práctica e innovación: aprender a emprender en el área de Bibliotecología y Ciencias de la Documentación. // Investigación Bibliotecológica. 28:63 (2014) 193-222.

Cubillo, Julio (1999). Cambio y continuidad en la biblioteca como organización gestora de conocimientos: reflexiones de un no-bibliotecario. // Cubillas, Julio (1999). Cambio y continuidad en la biblioteca como organización gestora de conocimientos: reflexiones de un nobibliotecario. Santiago de Chile: Naciones Unidas, Comisión Económica para América Latina y el Caribe, Centro Latinoamericano de Documentación Económica y Social, CEPAL/CLADES, 1999. ISBN 9213215711.15-23.

Harvey, Ana María; Muñoz, Daniel (2006). El género informe y sus representaciones en el discurso de los académicos. // Estudios filológicos. 41 (sep. 2006). http://minga online.uach.cl/scielo.php?script=sci_arttext\&pid=S0071$17132006000100008 \&$ Ing=es\&nrm=iso $(2015-03-20)$.

Instituto de Ciencias Nucleares (2009). Unidad de Información y Biblioteca. México: Instituto de Ciencias Nucleares, 2009. http://www.nucleares.unam.mx/icn2/index.p hp?option=com content\&view=article\&id=125\&ltemid $=144$ (2015-03-25).

Quijano Solís, Álvaro; Santiago Martínez, Alberto (2010). Sobre las competencias y los conocimientos del bibliotecario en el área de tecnologías de la información. // Revista Mexicana de Ciencias de la Información. 1:1 (2010) 54-62.

Enviado: 2015-04-07. Segunda versión: 2015-06-30. Aceptado: 2015-09-01. 